\title{
Influence of e-beam evaporation parameters on the microstructure of nanocrystalline and epitaxially grown Ti thin films
}

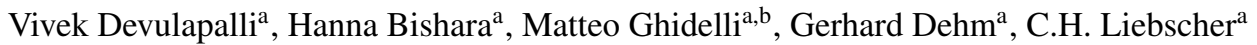 \\ ${ }^{a}$ Max-Planck-Institut für Eisenforschung GmbH, Max-Planck-Str. 1, D-40237 Düsseldorf, Germany \\ ${ }^{b}$ Laboratoire des Sciences des Procédés et des Matériaux (LSPM), CNRS, Université Sorbonne Paris Nord, 93430, Villetaneuse, France
}

\begin{abstract}
Titanium thin films were deposited on silicon nitride $\left(\mathrm{SiN}_{x}\right)$ coated $\mathrm{Si}, \mathrm{NaCl}$, and sapphire substrates varying the deposition conditions using e-beam evaporation to investigate thin film growth modes. The microstructure and texture evolution in dependence of substrate, deposition rate, film thickness, and substrate temperature were studied using X-ray diffraction, electron backscatter diffraction, and transmission electron microscopy. Thin films obtained on $\mathrm{SiN}_{x}$ and $\mathrm{NaCl}$ substrates were nanocrystalline, while the films deposited on sapphire transformed from nanocrystalline to single crystalline at deposition temperatures above $200^{\circ} \mathrm{C}$. Predominantly, a surface plane orientation of (0002) was observed for the single crystalline films due to the minimization of surface energy. The orientation relationship of epitaxial single crystalline films grown on C-plane sapphire substrate is found to be (0002) ${ }_{T i}\left\|(0006)_{\text {Sapphire }},\langle 11 \overline{2} 0\rangle_{T i}\right\|\langle 03 \overline{3} 0\rangle_{\text {Sapphire. }}$ In this orientation relationship, both the total surface and strain energy of the film are minimized. The results were complemented by resistivity measurements using the four-point probe method reporting an increase from $\sim 60 \mu \Omega \mathrm{cm}$ to $\sim 95 \mu \Omega \mathrm{cm}$ for single crystalline and nanocrystalline films, respectively.
\end{abstract}

\section{Introduction}

Titanium (Ti) is a commercially extensively used material ${ }^{33}$ due to its high strength to weight ratio and biocompatibility ${ }_{35}^{34}$ [1, 2]. Apart from its conventional bulk applications, there are emerging advancements to use it in microelectromechanical systems (MEMS) devices and hydrogen storage applications [3, 4, 5]. It is also used as an adhesive bonding layer to grow films of Pt, $\mathrm{GaN}$, and $\mathrm{Cu}$ thin films [6, 7, 8]. These applications demand the film to have a preferred microstructure such as nanocrystallinity for high strength and fatigue resistance [9].

Nanocrystalline Ti films have been reported on $\mathrm{NaCl}$ [10], ${ }^{42}$ which is a water-soluble substrate. It is extensively used as a growth substrate or as an intermediate sacrificial film for ul- ${ }^{4}$ tra-thin metallic films. It has a wide variety of applications like transfer-printing, nanotexturing, and fabrication of metallic nanostructures for transparent flexible electrodes [11]. Likewise, due to its cubic symmetry, $\mathrm{SiN}_{x}$ is a good contender for nanocrystalline Ti films. It has massive commercial importance as a powerelectronics substrate due to its high mechanical strength [12]. Furthermore, sapphire is a good choice as a substrate for de- ${ }^{51}$ positing epitaxial thin films due to its hexagonal symmetry[13]. Epitaxy is the oriented growth of target material on top of a ${ }^{5}$ substrate surface. Highly epitaxial films tend towards single- ${ }^{54}$ crystal-like behaviour, which is of great interest especially for ${ }^{55}$ next-generation nano- and power electronics, optical and mag- ${ }^{56}$ netic devices [14]. Due to the allotropic transitions, solidifica- 57 tion from the melt-pool is not a viable way to obtain single crys- 58 talline (SC) Ti. For applications such as coating medical devices ${ }^{59}$ with Ti to improve their biocompatibility and wear resistance, 60 epitaxial films to obtain a SC surface is highly attractive. There- 61 fore, the microstructure of Ti films on all these substrates is ex- 62 amined in this study.

Apart from the deposition parameters, the lattice matching, symmetry of surface planes, substrate miscut, and the planes forming terraces and steps on the substrate surface [15] are variables that can alter the orientation and morphology of thin films, while maintaining substrate chemistry. The adaption of an orientation relationship (OR) between a substrate and a film is a complex phenomenon [16]. Even with a known substrate surface symmetry and chemical composition, it is in most cases not possible to predict the preferred OR [17]. Although the (0002) plane of Ti has the lowest surface energy, making it the dominant surface plane, Ti films have been reported to grow with at least six other surface plane orientations [18].

The effect of deposition parameters on Ti thin films deposited using DC sputtering have been investigated earlier, which are predominantly reported to be nanocrystalline [19, 20, 21]. However, when using e-beam evaporation a range of microstructures and orientations have been observed. For depositions made on sapphire substrate, (1010) and (1120) SC films were observed when deposited at a very high rate of $350 \AA / s$ [22]. On the contrary, (0002) SC films were obtained with a growth rate of $20 \AA / s[13] .6 \AA / s$ was stated to be the lower limit to obtain a SC film [13], but Dehm et al. [23] reported SC Ti at much lower deposition rates of $0.15 \AA / s$ at substrate temperatures $\left(\mathrm{T}_{d e p}\right)$ ranging from $373 \mathrm{~K}$ to $473 \mathrm{~K}$ for up to $100 \mathrm{~nm}$ thick films. Further lowering of the deposition rate to $\sim 0.03 \AA / s$ lead to a polycrystalline film with 3D island growth [24]. Interestingly, the reduction of $\mathrm{Al}_{2} \mathrm{O}_{3}$ by formation of $\mathrm{Ti}_{3} \mathrm{Al}$ at the interface in sputter deposited Ti at room temperature (RT) with a deposition rate of $0.4 \AA / s$ has also been reported [25]. Furthermore, with similar deposition conditions on $\mathrm{NaCl}$ substrates, co-existing 
hcp and face-centered cubic (fcc) grains, as well as the entire 56 film being solely nanocrystalline hep grains, have been doc- 57 3 umented [26, 10]. Overall, these studies illustrate that a wide 58 4 range of films can be obtained on sapphire and $\mathrm{NaCl}$. This ne- 59 cessitates a thorough investigation of the effect of deposition 60 parameters on thin film microstructure.

In this work, $\mathrm{Ti}$ thin films were deposited on $\mathrm{NaCl}$, silicon 62 nitride $\left(\mathrm{SiN}_{x}\right)$ on $\mathrm{Si}(100)$, and sapphire substrates using e-beam 63 evaporation. The $\mathrm{T}_{d e p}$ was varied from $\mathrm{RT}$ to $400^{\circ} \mathrm{C}$ and the 64 growth rate was altered between $0.25 \AA / s$ to $2.5 \AA / s$. The film ${ }_{65}$ thicknesses were on the order of $100-300 \mathrm{~nm}$. The microstruc- 66 ture and OR between the film and substrate were studied using 67 electron backscatter diffraction (EBSD) and transmission elec- 68 tron microscopy (TEM). The first section presents the textured 69 nanocrystalline films obtained on $\mathrm{SiN}_{x}$ and $\mathrm{NaCl}$ substrates, 70 followed by details of single-crystalline films obtained on sap- 71 phire. The role of the substrate on the resulting microstructures 72 and ORs is discussed. Electrical resistivity measurements indi- 73 cated a limited change of resistivity within the SC films based 74 on their thickness but a steep increase in the nanocrystalline 75 film.

\section{Experimental}

Ti pellets $(99.995 \%, 1 / 4$ "dia.) were purchased from Kurt J. 80 Lesker Company to be used as the target. The films were de- 81 posited in a BesTeck PVD cluster (MPIE, Düsseldorf) on $\mathrm{NaCl}_{82}$ (100), $\mathrm{SiN}_{x}$ on silicon (100) and sapphire C-plane (0002), C- 8 plane with a $2^{\circ}$ miscut along [11̄20], sapphire A-plane $(11 \overline{2} 0)_{84}$ and R-plane (1102) substrates. The deposition conditions are 85 listed in Table 1. $\mathrm{SiN}_{x}$ and sapphire substrates were obtained 86 from Siegert Wafer $\mathrm{GmbH}$. In the $\mathrm{SiN}_{x}$ substrate, the $50 \mathrm{~nm}$ ni- 87 tride layer on the $\mathrm{Si}(100)$ substrate acts as a diffusion barrier ${ }_{88}$ and prevents the reduction of $\mathrm{Si}$ with $\mathrm{Ti}$ at higher temperatures. The $\mathrm{SiN}_{x}$ substrate was heated to $500^{\circ} \mathrm{C}$ for $30 \mathrm{~min}$ before the deposition to clear off adsorbates and improve film adhesion. ${ }^{89}$ The substrate was then cooled down to $200^{\circ} \mathrm{C}$ for deposition. ${ }_{90}$ The $\mathrm{NaCl}$ substrate was cleaved in air right before the deposition to obtain clean (100) surface planes. Sapphire wafers were ${ }^{9}$ one side epi-polished having a surface roughness of $\leq 0.3 \mathrm{~nm}$. These substrates were used in the as-received state with no ad- ${ }^{93}$ ditional surface treatments required.The deposition chamber ${ }^{94}$ was pumped down to $2 \times 10^{-8} \mathrm{mbar}$ for all the depositions. The substrate to target distance was $\sim 250 \mathrm{~mm}$. The e-beam current was tuned to the values ranging from 50 to $150 \mathrm{~mA}$ which was calibrated using a quartz microbalance and constantly monitored during the deposition. To double-check the calibration, the actual deposition rate was calculated post-deposition using film thickness and deposition duration.

First, the surface of the deposited films was subsequently ${ }^{102}$ examined by using a Zeiss Axio Imager light optical micro- ${ }^{103}$ scope (LOM) to detect cracks or other visible defects. The ${ }^{104}$ global film microstructure was determined by X-ray diffraction ${ }^{105}$ a Huber 4 circle goniometer using $\mathrm{Cu}-\mathrm{K} \alpha(\lambda=1.54 \AA)$ and $^{107}$ Co-K $\alpha(\lambda=1.78 \AA)$ radiation and a scintillation detector to ver- ${ }^{108}$ ify the purity of the films and to measure the macro-texture. ${ }^{109}$ (XRD) using a Seifert ID3003 diffractometer equipped with ${ }^{106}$
XRD was performed for a $2 \theta$ range of 30 to $90^{\circ}$ with a point focused beam of $1 \times 3 \mathrm{~mm}$ diameter. Grazing incidence XRD (GIXRD) was used with a $1^{\circ}$-incidence angle to avoid diffraction from the single crystal substrates.

The local surface morphology and film orientation was determined by scanning electron microscopy (SEM) and EBSD in a Thermo Fisher Scientific Scios2HiVac dual-beam SEM. For films deposited on $\mathrm{NaCl}$ substrate, the substrate was dissolved in distilled water and then picked up using $3 \mathrm{~mm}$ diameter molybdenum TEM grids. The grids containing the film can be directly placed inside the TEM holder after drying for several hours in the air. For films deposited on sapphire, a standard lift-out technique in a focused ion beam (FIB) instrument (Scios2HiVac) with $\mathrm{Ga}^{+}$-ion source was used to extract TEM lamella of $<100 \mathrm{~nm}$ thickness. The beam current was gradually reduced in several steps starting from $1 \mathrm{nA}$ at $30 \mathrm{kV}$ for coarse milling to eventually $27 \mathrm{pA}$ at $2 \mathrm{kV}$ for final polishing. All TEM work was done using an image aberration-corrected Titan Themis 80-300 (Thermo Fischer Scientific) TEM. Selected area diffraction patterns (SADP) were obtained by inserting a 10 $\mu \mathrm{m}$ aperture. High-resolution transmission electron microscopy (HRTEM) observations were done under negative $\mathrm{C}_{s}(-10 \mu \mathrm{m})$ conditions using an accelerating voltage of $300 \mathrm{kV}$.

The electrical resistivity of the thin films was determined by adapting the Van der Pauw method [27] on $1 \mathrm{~cm} \times 1 \mathrm{~cm}$ pieces cut from the wafer after deposition. Four contacts were positioned on the top surface close to the corners of the sample using a LINKAM HFS-600 system. A direct current (DC) of $0.01 \mathrm{~A}$ is applied by a current generator (Keithly 6221) in the form of $10 \mathrm{~ms}$ pulses. The consequent voltage is measured at half-time of the pulse duration employing a voltmeter (Keithly 2182A). A series of 200 pulses are applied for increased accuracy.

\section{Results and Discussion}

\subsection{Deposition on $\mathrm{SiN}_{x}$ and $\mathrm{NaCl}$}

At a deposition rate of $2.5 \AA / \mathrm{s}$, a $75 \mathrm{~nm}$ thick Ti film was deposited on $\mathrm{SiN}_{x}$ substrate heated to $200^{\circ} \mathrm{C}$. According to LOM, a smooth surface with no visible cracks or porosity was obtained (Sup. Fig. 1). The polycrystalline nature of the film is revealed by the presence of all diffraction planes in the GIXRD data shown in red in Fig. 1. From the full width at half maximum (FWHM) of the most intense Ti peak and using the Scherrer equation [28], the average crystallite size was estimated to be 20 to $30 \mathrm{~nm}$ along the surface normal direction. The peaks were symmetrical with the $2 \theta$ peak positions coinciding with that of bulk $\alpha$-Ti [29], which indicates that global residual stresses in the film are negligible. However, a preferred (1010) texture was observed.

To explain the observed texture, the total free energy of a thin film can be calculated by the sum of the surface and strain energy [30] which the system tries to minimize during deposition. The surface energy is determined by the coordination number or atomic density of the respective lattice planes. In $\mathrm{Ti}$, the (0002) plane has the lowest surface energy $\left(2.8 \mathrm{~J} / \mathrm{m}^{2}\right)$, 
Table 1: List of deposited films using e-beam deposition with their deposition parameters.

\begin{tabular}{|c|c|c|c|c|c|}
\hline Sample No. & Substrate & Deposition temperature $\left({ }^{\circ} \mathrm{C}\right)$ & Thickness (nm) & Rate $(\AA / s)$ & Microstructure \\
\hline Sil & $\mathrm{Si}_{3} \mathrm{~N}_{4}(100)(50 \mathrm{~nm})$ & $200^{\circ} \mathrm{C}$ & 75 & 2.5 & $\mathrm{NC}$ \\
\hline N1 & $\mathrm{NaCl}(100)$ & RT & 90 & 0.25 & $\mathrm{NC}$ \\
\hline $\mathrm{N} 2$ & $\mathrm{NaCl}(100)$ & RT & 90 & 0.8 & $\mathrm{NC}$ \\
\hline N3 & $\mathrm{NaCl}(100)$ & $200^{\circ} \mathrm{C}$ & 75 & 2.5 & $\mathrm{NC}$ \\
\hline S1 & Sapphire - C-plane (0001) & RT & 200 & 0.5 & $\mathrm{SC}$ \\
\hline $\mathrm{S} 2 *$ & Sapphire - C-plane (0001) & $300^{\circ} \mathrm{C}$ & 200 & 0.7 & $\mathrm{SC}$ \\
\hline S3 & Sapphire - C-plane (0001) & RT & 90 & 2.5 & $\mathrm{SC}$ \\
\hline S4 & Sapphire $-\mathrm{c}-2^{\circ}$ miscut & RT & 90 & 2.5 & $\mathrm{NC}$ \\
\hline S5 & Sapphire - A-plane (112̄0) & RT & 90 & 2.5 & $\mathrm{NC}$ \\
\hline S6 & Sapphire - R-plane (11̄02) & RT & 90 & 2.5 & $\mathrm{NC}$ \\
\hline S7 & Sapphire - C-plane (0001) & $200^{\circ} \mathrm{C}$ & 300 & 2.5 & $\mathrm{SC}$ \\
\hline S8 & Sapphire $-c-2^{\circ}$ miscut & $200^{\circ} \mathrm{C}$ & 300 & 2.5 & $\mathrm{SC}$ \\
\hline S9 & Sapphire - A-plane (112̄0) & $200^{\circ} \mathrm{C}$ & 300 & 2.5 & $\mathrm{SC}$ \\
\hline S10 & Sapphire - R-plane (11̄02) & $200^{\circ} \mathrm{C}$ & 300 & 2.5 & $\mathrm{NC}$ \\
\hline S11 & Sapphire - C-plane (0001) & $400^{\circ} \mathrm{C}$ & 300 & 2.5 & $\mathrm{SC}$ \\
\hline S12 & Sapphire $-\mathrm{c}-2^{\circ}$ miscut & $400^{\circ} \mathrm{C}$ & 300 & 2.5 & $\mathrm{SC}$ \\
\hline S13 & Sapphire - A-plane (11̄̄0) & $400^{\circ} \mathrm{C}$ & 300 & 2.5 & $\mathrm{SC}$ \\
\hline S14 & Sapphire - R-plane (1102) & $400^{\circ} \mathrm{C}$ & 300 & 2.5 & $\mathrm{SC}$ \\
\hline
\end{tabular}

RT - Room temperature, NC - Nanocrystalline, SC - Single-crystalline, * - Deposited using Ar-ion sputtering,

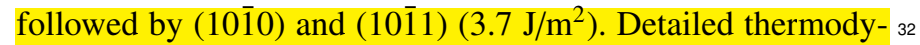
namic calculations of the surface energy using their planar den- 3 sities have been reported earlier [18, 31]. The strain energy, on 34 the other hand, is defined by the film's strain and the anisotropy 35 of elastic modulus [18]. The elastic anisotropy of Ti results in ${ }_{36}$ a 30\% lower elastic modulus for the [1010] than the [0002] di- 37 rection [18]. As a result, a competing growth of these three planes is observed, resulting in the film's overall free energy being minimized [17]. The lower strain energy of the (1010) orientation seems to be responsible for the observed texture in the film grown on $\mathrm{SiN}_{x}$.

The film deposited on $\mathrm{NaCl}$ (100) under the same conditions $\left(\mathrm{T}_{d e p}=200^{\circ} \mathrm{C}\right.$, Rate $=2.5 \AA / \mathrm{s}$, thickness $\left.=75 \mathrm{~nm}\right)$, was observed using LOM to be severely buckled (Sup. Fig. 2), indicating large residual compressive stresses in the film. The thermal expansion coefficient of $\mathrm{NaCl}$ is an order of magnitude higher than $\mathrm{Ti}$ [32], which promotes the evolution of large compressive stresses in the film upon cooling from $\mathrm{T}_{d e p}$ to RT leading to buckling. The GIXRD pattern shown in Fig. 1 confirms the polycrystalline nature of the film albeit with a (1011) texture. The revealed texture matches well with that observed by Campbell et al. [26]. This can be explained by the relatively low surface energy of the (1011) plane, as discussed above, making it one of the favoured surface normal planes. In addition, the (10ī1) orientation leads to lower lattice misfit than (0002) orientation. $\mathrm{NaCl}$ has a lattice constant of $5.64 \AA$, and the Ti (1011) plane has a cell size of $2.95 \times 10.66 \AA^{2}$. Simply based on the geometry, the misfit between the film and 38 substrate can be calculated by superimposing the $\mathrm{Ti}(10 \overline{1} 1)$ plane $_{39}$ on the $\mathrm{NaCl}$ (100) plane. The OR leading to the lowest misfit 40 can hence be calculated as:

$$
\delta=\left(1-\frac{d_{f}}{d_{s}}\right)
$$

where $\mathrm{d}_{s}$ is the lattice spacing of the substrate, $\mathrm{d}_{f}$ the lattice spacing of the film plane. The lowest misfit of $5.49 \%$ is achieved for $(10 \overline{1} 1)_{T i} \|(001)_{\mathrm{NaCl}}$ when using the domain matching epitaxy (DME) [33] with a $1 / 2$ domain matching between $\mathrm{Ti}$ and $\mathrm{NaCl}$. This describes the (1011) texture that is observed.

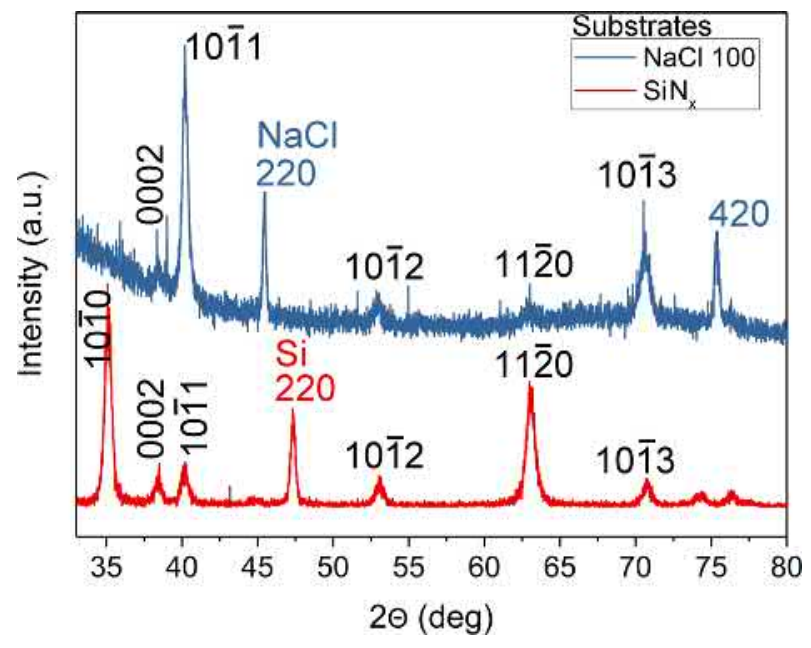

Figure 1: Grazing-Incidence XRD $(\mathrm{Cu}-\mathrm{K} \alpha$ source $)$ of a $75 \mathrm{~nm}$ thick Ti thin film deposited at $200^{\circ} \mathrm{C}$ on $\mathrm{SiN}_{x}$ (100) (sample \#Si1) and $\mathrm{NaCl}$ (100) (sample \#N3). Both films exhibit a polycrystalline microstructure. The film deposited on $\mathrm{SiN}_{x}$

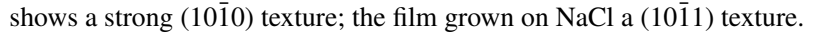

In Fig. 2a the cross-sectional bright-field TEM (BF-TEM) image of the film grown on $\mathrm{SiN}_{x}$ substrate confirms the nanocrystalline microstructure and the absence of any reaction between the film and the substrate. The grain size of $20 \pm 5 \mathrm{~nm}$ is in the same range as estimated from the XRD data above. The corresponding diffraction pattern shown in Fig. $2 \mathrm{~b}$ also verifies the 
film's nanocrystalinity and hcp crystal structure.

Furthermore, for all films grown on $\mathrm{NaCl}$, TEM was used ${ }_{31}$ to examine the nanocrystalline grain structure and to determine 32 the impact of deposition rate and $\mathrm{T}_{d e p}$ on the film microstruc- ${ }_{3}$ ture. Fig. 2c shows a representative plane view BF-TEM im- 34 age of sample \#N1 (nanocrystalline film on $\mathrm{NaCl}$ at RT; de- 35 position rate $=0.25$ The grain size was measured by the line ${ }_{36}$ intercept method [34] to be $\sim 54 \pm 13 \mathrm{~nm}$. The film adopts 37 the $\alpha$-Ti (hcp) structure as verified from the diffraction rings 38 in Fig. 2d.Although the film produced ring patterns, a pre- 39 ferred (1011) texture is observed, which is identical to what 40 the XRD revealed above. The surface diffusivity of condens- 41 ing Ti atoms on the substrate surface is considerably lower 42 at RT compared to elevated temperatures. According to the 43 structural zone model, the lack of sufficient thermal energy to activate surface diffusion of adatoms results in the formation of nanocrystalline grains at RT [35].
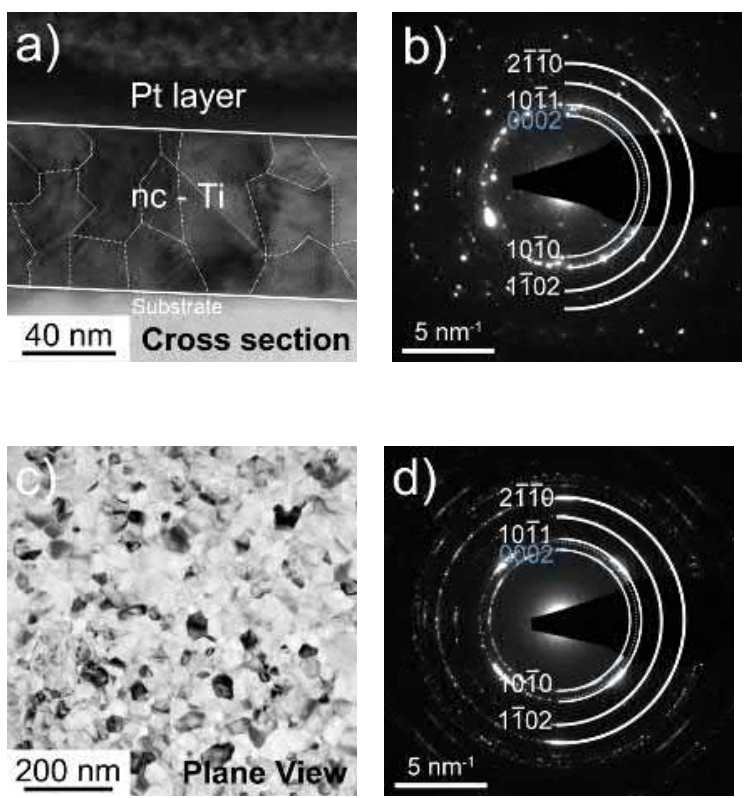

Figure 2: TEM bright field image showing the microstructure of the Ti thin film deposited (a) at $200^{\circ} \mathrm{C}$ on $\mathrm{SiN}_{x}$ substrate (cross section, sample \#Si1) and (c) at $\mathrm{RT}$ on $\mathrm{NaCl}$ (100) substrate (plane view, sample \#N1), with respective ${ }^{61}$ selected area diffraction patterns in (b) and (d) verifying the nanocrystalline ${ }^{62}$ hcp $\alpha$-Ti structure.

The BF-TEM images shown in Fig. 3 a and Fig. $3 \mathrm{c}$ are ${ }^{65}$ taken from sample \#N2 on $\mathrm{NaCl}(100)$ at RT; deposition rate ${ }^{66}$ $=0.8 \AA / \mathrm{s})$ and sample \#N3 on $\mathrm{NaCl}(100)$ at $200^{\circ} \mathrm{C}$; deposi- ${ }^{67}$ tion rate $=2.5 \AA / \mathrm{s}$ ) respectively. The average grain size of ${ }^{68}$ both films was determined to be $\sim \mathbf{3 1} \pm \mathbf{1 0} \mathrm{nm}$ and $\sim \mathbf{5 3} \pm{ }^{69}$ $18 \mathrm{~nm}$, as shown in Table 2 . The grain size decreases from ${ }^{70}$ $54 \pm 13 \mathrm{~nm}$ in \#N1 to $31 \pm 10 \mathrm{~nm}$ in \#N2 with increasing ${ }^{71}$ deposition rate, both at RT. The smaller grain size stems ${ }^{72}$ from the increase in nucleation rate. The increased nucle- ${ }^{73}$ ation events are a direct consequence of the lower mean free ${ }^{74}$ path due to the higher flux of adatoms at higher deposition ${ }^{75}$ rates. This observation is contrary to the general obser- ${ }^{76}$ vation that an increase in deposition rate increases grain size [36]. The latter is valid only at higher deposition temperatures when growth is not limited by surface diffusion [37]. Because these depositions were performed at RT, surface diffusion is severely limited. On the other hand, for sample \#N3 (Fig. 3c), the deposition temperature was increased from RT to $200^{\circ} \mathrm{C}$. The increase in substrate temperature results in a higher surface diffusivity leading to larger grain size. Assuming that the cleaved $\mathrm{NaCl}$ substrate surface played an identical role in the film growth in all cases, the observations indicate that lowering the deposition rate at $\mathrm{RT}$ and increasing the deposition rate at higher temperatures leads to an increase in grain size for $\mathrm{NaCl}(100)$ substrate.

Table 2: Grain size distribution of Ti thin films deposited on $\mathrm{NaCl}$ (100) by varying substrate temperature and deposition rate

\begin{tabular}{|cccc|}
\hline Film & $\mathrm{T}_{d e p}\left({ }^{\circ} \mathrm{C}\right)$ & Rate $(\AA / s)$ & Grain size $(\mathrm{nm})$ \\
\hline Si1 & $200^{\circ} \mathrm{C}$ & 2.5 & $20 \pm 5$ \\
$\mathrm{~N} 1$ & $\mathrm{RT}$ & 0.2 & $54 \pm 13$ \\
$\mathrm{~N} 2$ & $\mathrm{RT}$ & 0.8 & $31 \pm 10$ \\
$\mathrm{~N} 3$ & $200^{\circ} \mathrm{C}$ & 2.5 & $53 \pm 18$ \\
\hline
\end{tabular}

The corresponding SADPs shown in Fig. 3b and Fig. 3d confirm the nanocrystalline nature of the films. The intensity of diffraction spots is restricted to only certain orientations going from film \#N1 to \#N3 as seen in Fig. 2d, Fig. 3b and Fig. 3d respectively. Hence, an increasing degree of (1011) texture is observed on increasing both deposition rate and $\mathrm{T}_{\text {dep }}$.

It is known that water is adsorbed on the surface of alkali halides [38]. Based on the humidity of the environment the (100) surface of $\mathrm{NaCl}$ is most likely covered with 0.5 monolayer of water at RT. Thus, the surface partly resembles an amorphous state [39]. For amorphous substrates, the surface energy $\gamma_{i}$ is not expected to change with the in-plane rotation, which results in an equal probability of grains with all possible in-plane rotations to grow [40]. Since the substrate was cleaved in air in our experiments, we can conclude that water is physisorbed on its surface and the polycrystalline film deposited on $\mathrm{NaCl}$ at $\mathrm{RT}$ should exhibit all in-plane rotations. However, the minimization of surface and interface energy during the pre-coalescence stage of the film growth plays a major role in deciding the texture of the film [40]. Hence, a weak (1011) texture is observed in Fig.2d. In film $\#$ N2, the increased deposition rate leads to preferred growth of low energy planes promoting the (1011) texture. Once the deposition temperature is raised, the physisorbed water layer evaporates from the $\mathrm{NaCl}$ surface. Hence, in film \#N3, where the substrate was heated to $200^{\circ} \mathrm{C}$ during deposition, the surface anisotropy of $\mathrm{NaCl}$ comes into play leading to a much stronger (1011) texture. This exhibits the control in microstructure that is made available by alteration of the deposition parameters. All the four films discussed in the preceding section are nanocrystalline. As a result, these findings pave the way for better-informed deposition of nanocrystalline Ti, which is known for its exciting properties such as high strength 
and corrosion resistance [41].
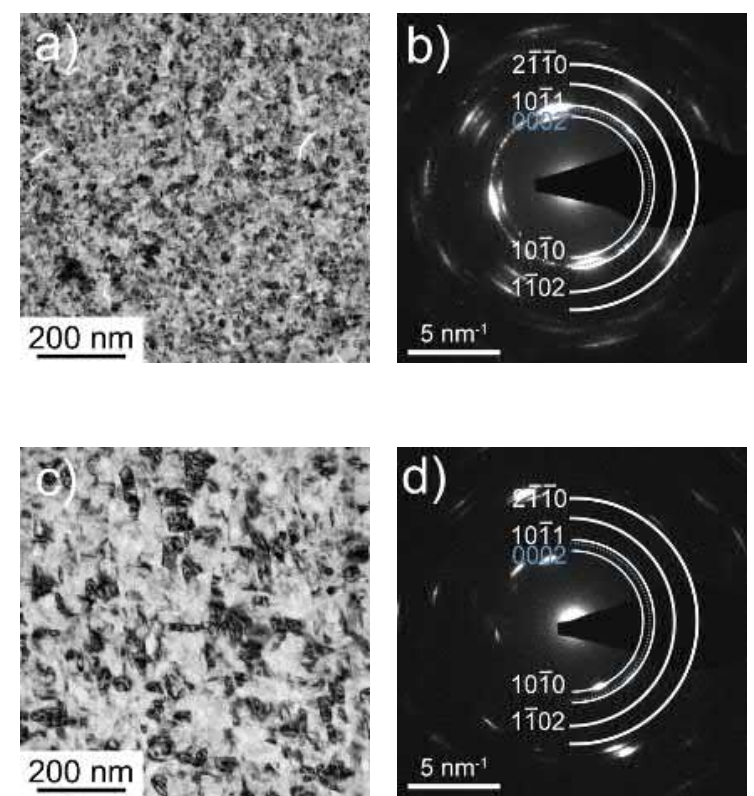

Figure 3: Plane view TEM bright field image of a Ti thin film deposited at (a) $0.8 \AA / \mathrm{s}$ deposition rate at RT (sample \#N2) and (b) $2.5 \AA / \mathrm{s}$ deposition rate at $200^{\circ} \mathrm{C}$ on $\mathrm{NaCl}(100)$ substrate (sample N3). The corresponding selected area diffraction patterns are shown in (b) and (d) respectively, both exhibiting a strong (1011) texture.

poly-/nanocrystalline as has been reported in several other materials grown on sapphire [43, 44]. However, a (0002) texture is observed in all three cases similar to the C-plane substrate which can be explained by its low surface energy.
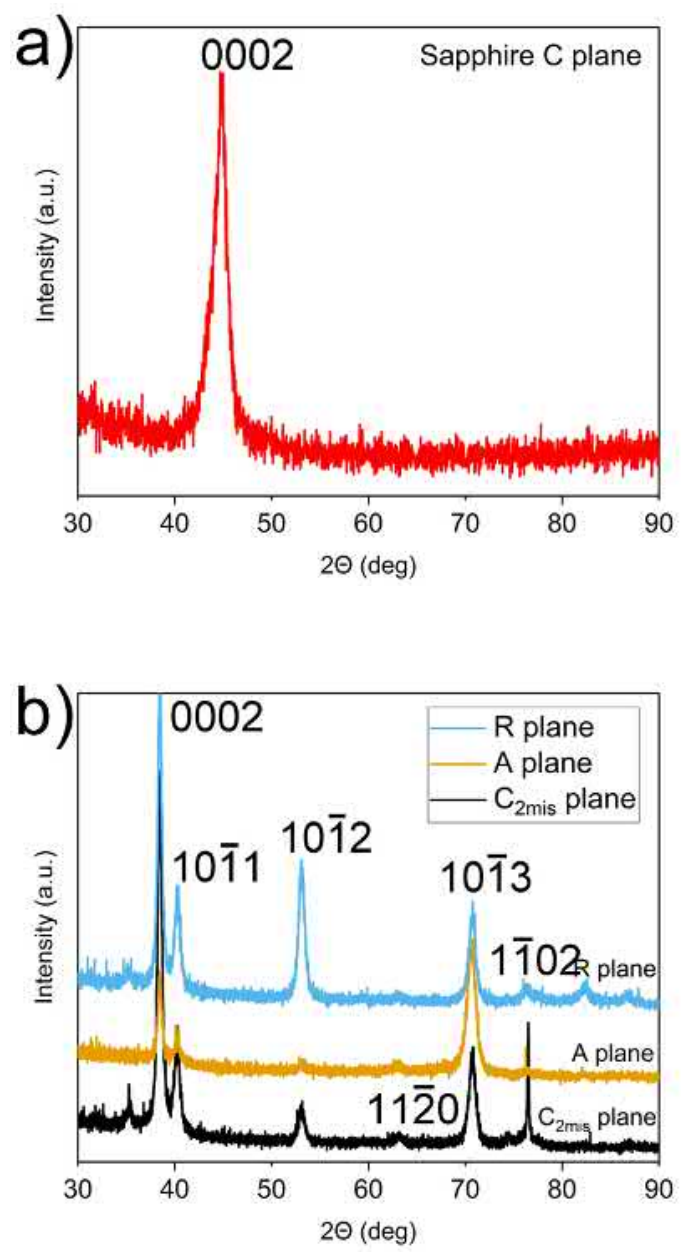

Figure 4: Grazing-Incidence XRD of $90 \mathrm{~nm}$ thick Ti thin films deposited at RT on (a) C-plane sapphire with only the (0002) peak present (Co-K $\alpha$ source), (b) C-plane with a $2^{\circ}$ miscut $\left(\mathrm{C}_{2 m i s}\right)$, A-plane and R-plane sapphire displaying multiple diffraction peaks $(\mathrm{Cu}-\mathrm{K} \alpha$ source)

Further batches of depositions using the same set of sapphire substrates were performed at higher deposition temperatures to study the influence of $\mathrm{T}_{d e p}$ on film microstructure. The deposition on C-plane sapphire, $\mathrm{C}_{2 m i s}$ and A-plane at $200^{\circ} \mathrm{C}$ (samples \#S7, \#S8 and \#S9) resulted in highly epitaxial singlecrystalline films. They were deposited after holding the substrates at $\mathrm{T}_{d e p}$ for $\sim 1 \mathrm{~h}$ to ensure uniform temperature. The holding period results in desorption of water and any organic/ volatile impurities from the substrate surface. An epi-polished sapphire substrate surface is known to exhibit surface steps [45]. Additionally, the increase of $\mathrm{T}_{d e p}$ results in an increased surface diffusion of adatoms. The islands which nucleate on the atomic steps in the $\mathrm{C}_{2 m i s}$ substrate are hence expected to have fixed orientation relationship with surface steps and better interconnections between neighboring grains at higher $\mathrm{T}_{d e p}$, which leads to a single dominant orientation [43]. A similar effect is 
observed in A-plane sapphire which leads to the ease of SC film formation. However, the film remained polycrystalline for the R-plane sapphire substrate at $200^{\circ} \mathrm{C}$.

There are three ways epitaxial films deposited on sapphire substrates can grow. When the bond between the adatom and the substrate is strong, a layer-by-layer deposition called Frank-van der Merwe (FM) mode can occur [46]. The Volmer-Weber ( VW) growth mode is caused by a stronger bond between the adatoms, resulting in their threedimensional clusters to coalesce and form a continuous film. A combination of the two, where clusters form on top of a few monolayers is termed Stranski-Krastanov (SK) growth mode [46]. Which of these growth modes is dominant is determined by the rivalry between the bare substrate's surface energy $\left(\gamma_{s v}\right)$, the interface energy between the substratefilm $\left(\gamma_{s f}\right)$, and between the film-vapor $\left(\gamma_{f v}\right)$. A large misfit system like in the above films leads to a high $\gamma_{s f}$ value, which results in a significantly lower barrier to nucleate clusters, favouring the VW growth mode. As a result of the formation of several tiny nuclei at RT, a nanocrystalline film was found in all but one of the cases. The only exception was C-plane sapphire, which was SC as previously discussed.

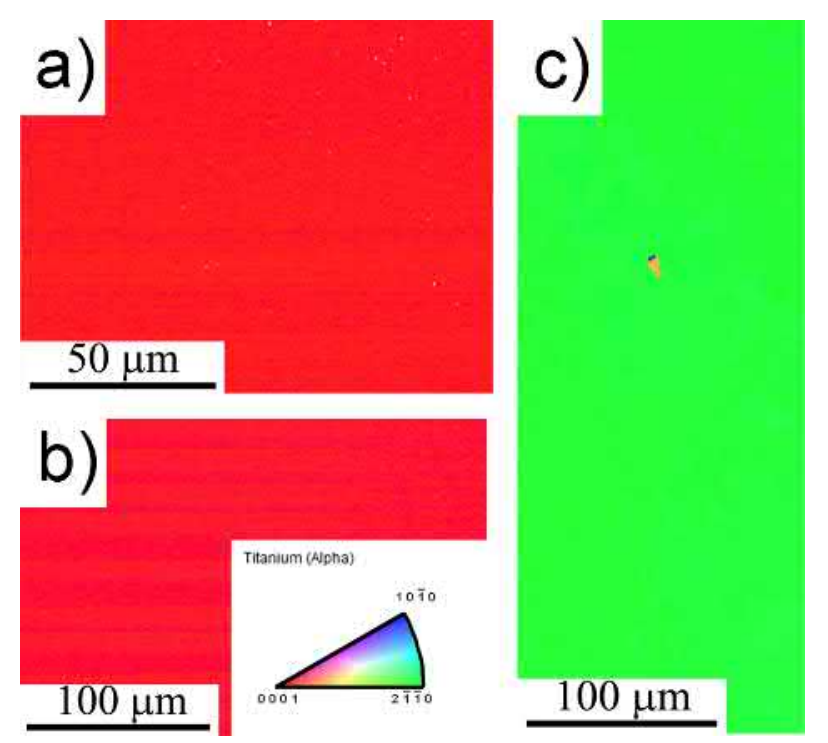

Figure 5: Inverse pole figure (IPF) obtained from EBSD of Ti thin films ${ }^{50}$ deposited at $400^{\circ} \mathrm{C}$ on (a) $\mathrm{C}_{2 m i s}$-plane (b) A-plane and (c) R-plane sapphire ${ }^{51}$ at low magnification to display uniform orientation over the entire wafer. 52

To verify the observations and to explain the anomalous be- 54 4 haviour of R-plane sapphire, the substrate temperature was fur- 55 25 ther increased to $400^{\circ} \mathrm{C}$. This leads to a further increased mobil- 56 26 ity of adatoms resulting in SC film formation on all the sapphire ${ }_{57}$ 27 substrate orientations (samples \#S11, \#S12, \#S13 and \#S14). 58 EBSD scans of the film surfaces revealed no change in orien- 59 tation. The scans were observed in both transverse and planar so viewing direction using TSL-OIM software to confirm the ab- 60 sence of grain boundaries even with a $1^{\circ}$ angular deviation. The ${ }_{61}$ 32 film deposited on C-plane (not shown), $\mathrm{C}_{2 m i s}$ and A-plane all ${ }_{62}$ 3 demonstrated the same preferred $(0002)_{T i}$ orientation as shown ${ }_{63}$ 4 in Fig. 5 a and b. The orientation of the film on C-plane and ${ }_{64}$

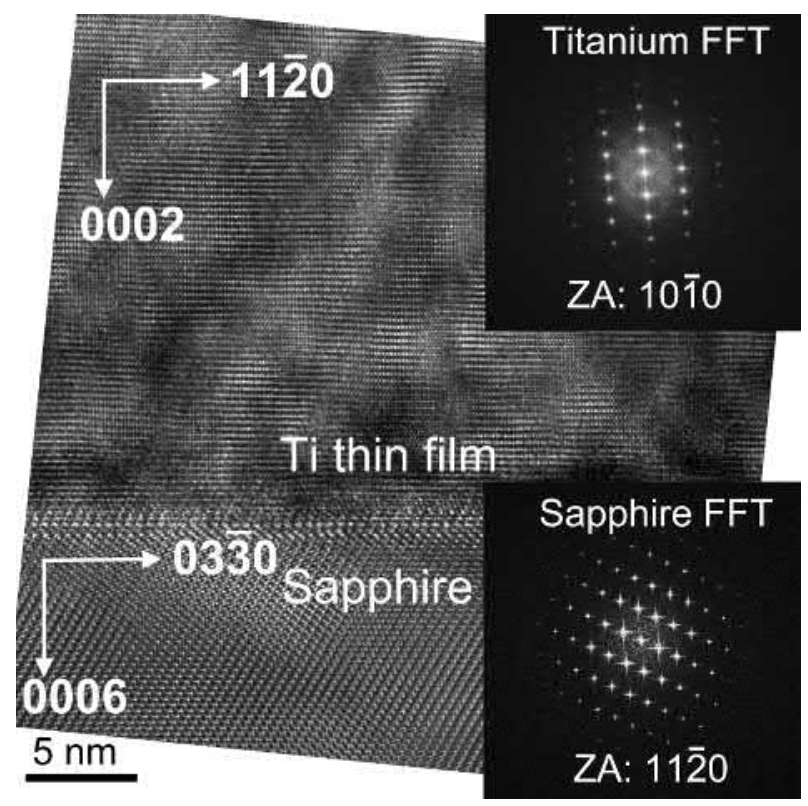

Figure 6: HRTEM image of cross-section of Ti thin film deposited on C-plane sapphire shows a $90 \mathrm{~nm}$ thick Ti film deposited using e-beam evaporation at RT (sample \#S3) observed in [112̄0] zone axis orientation of the sapphire substrate. The insets show fast Fourier transforms (FFTs) of the film and substrate, respectively, which are used to determine the ORs OR between the SC film and the C-plane sapphire substrate in
the sample \#S3 (deposited using e-beam evaporation at RT). As shown in Fig. 6, accommodating a 7.58\% misfit [48], the film was found to have (0002) ${ }_{T i}\left\|(0006)_{\text {Sapphire }},\langle 11 \overline{2} 0\rangle_{T i}\right\|$ $\langle 03 \overline{3} 0\rangle_{\text {Sapphire }}$ OR in agreement with [23]. Sup. Fig. 3 shows the sample \#S2 deposited with $\mathrm{Ar}$-ion sputtering at $200^{\circ} \mathrm{C}$ exhibits the same OR. This demonstrates that the OR between Ti and sapphire C-plane is independent of the deposition method.

\subsection{Resistivity measurements}

Ti thin films can be used in micro-electronic miniaturised circuits, electrical contacts and sputter ion pumps. These applications require a control of the electrical resistivity of the thin films. Based on the thickness, deposition parameters, chemical 
purity of the target and microstructure of the film, the resis- 35 tivity values can largely vary [49]. As seen in the Fig. 7, for 36 the films deposited on C-plane sapphire, the thinner film ${ }_{37}$ (sample \#S3), with thickness of $90 \mathrm{~nm}$, shows a higher re- 38 sistivity of $\sim 70 \mu \Omega \mathrm{cm}$. This increased resistivity can be ${ }_{39}$ attributed to increased surface scattering. For the $300 \mathbf{~ n m} 40$ thick SC films (sample \#S7 and \#S11) resistivity remains 41 uniform at $\sim 60 \mu \Omega \mathrm{cm}$, which is slightly higher than the ${ }_{42}$ pure bulk single crystal values of $\mathrm{Ti}(\sim 40 \mu \Omega \mathrm{cm})[\mathbf{5 0}, 22]_{43}$ which can also be attributed to surface scattering. The val- ${ }_{44}$ ues measured here are in agreement with other epitaxial $\mathrm{Ti}_{45}$ films [22], and are much lower than the polycrystalline films ${ }_{46}$ reported in literature [51, 50, 52]. Apart from the surfaces, ${ }_{47}$ grain boundaries can also act as scattering centers. Resulting ${ }_{48}$ from increased scattering from grain boundaries, the nanocrys- 49 talline film grown on sapphire A-plane at RT (sample S5) exhib- ${ }_{50}$ ited a much higher resistivity of $\sim 95 \mu \Omega \mathrm{cm}$, while the thicker ${ }_{51}$ films on A-plane sapphire show the same trend as seen for C- ${ }_{52}$ plane. As suggested by Bel'skaya et al. [53], the resistivity ${ }_{53}$ values of thin films can also be used as a measure of the impu- ${ }_{54}$ rity content, given the deposition conditions are same. Hence, ${ }_{55}$ the resistivity of $\sim 60 \mu \Omega \mathrm{cm}$ in the SC films indicates that im- ${ }_{56}$ purities play a minor role on resistivity.

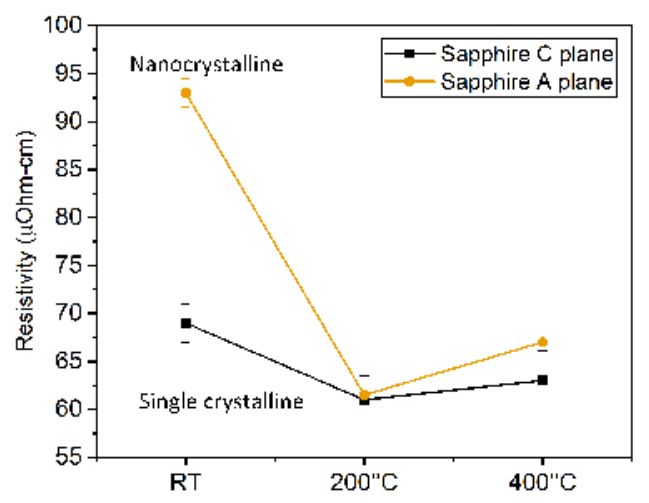

Figure 7: Resistivity values of films grown on C-plane sapphire and A-plane 7 at varying $\mathrm{T}_{\text {dep }}$. The only nanocrystalline film shows higher resistivity of $\sim 95 \mu \Omega \mathrm{cm}$ while all SC films show resistivity of $\sim 60 \mu \Omega \mathrm{cm}$

\section{Conclusion}

In this study titanium thin films were successfully deposited ${ }_{77}^{76}$ using e-beam evaporation on substrates of $\mathrm{SiN}_{x}, \mathrm{NaCl}$ and four ${ }_{78}$ orientations of sapphire. The substrate temperature and depo- 79 sition rate were varied in order to study their influence on mi- ${ }^{80}$ crostructure and texture. on $\mathrm{SiN}_{x}$. All depositions on $\mathrm{NaCl}$ substrate also led ${ }_{85}^{84}$ to nanocrystalline films. However, a difference in tex- 86 ture between the two was observed. The minimization ${ }^{87}$ of strain energy dominated for the film deposited on ${ }^{88}$
$\mathrm{SiN}_{x}$ substrate leads to a (1011) texture, while surface energy minimization promotes a $(10 \overline{1} 0)$ texture of all films deposited on $\mathrm{NaCl}(\mathbf{1 0 0})$. An increase of deposition rate resulted in a minor increase in the grain size. Nevertheless, all the films were nanocrystalline, which can be of great commercial interest for applications requiring high strength and corrosion resistance.

2. The film on C-plane sapphire grew as a single crystal with (0002) plane surface normal in all cases. The sixfold symmetry of the oxygen sub-lattice of sapphire and the low lattice misfit are considered to be responsible for the formation of a SC film even at RT. Using crosssection HRTEM imaging the orientation relationship was determined to be (0002) ${ }_{T i} \|$ (0006) Sapphire $_{\text {, }}\langle 11 \overline{2} 0\rangle_{T i} \|$ $\langle 03 \overline{3} 0\rangle_{\text {Sapphire }}$.

3. On C-plane sapphire with a $2^{\circ}$ miscut and A-plane, a nanocrystalline film grew at RT and SC films were obtained by heating the substrate to $200^{\circ} \mathrm{C}$. For the first time, a SC film was obtained on R-plane sapphire by heating the substrate to $400^{\circ} \mathrm{C}$.

4. The resistivity of the $90 \mathrm{~nm}$ thick SC film was $70 \mu \Omega \mathrm{cm}$ while $300 \mathrm{~nm}$ thick films displayed lower resistivity of $60 \mu \Omega \mathrm{cm}$ owing to reduced surface scattering with increasing film thickness. The nanocrystalline film exhibited higher resistivity of $95 \mu \Omega \mathrm{cm}$ because of additional scattering from grain boundaries.

These results pave the way to a controlled synthesis of Ti films with defined orientations having fundamental implications for micro-electronics and thin film industry.

\section{Acknowledgement}

VD and CHL acknowledge funding from the KSB Stifftung. $\mathrm{HB}$ and GD acknowledge funding from the European Research Council (Grant no. 787446-GB-CORRELATE). The authors would like to thank Mr. Benjamin Breitbach for conducting the X-ray diffraction experiments. The assistance of Mr. Dennis Klapproth and Mr. Andrea Brognara during film deposition is also gratefully acknowledged.

1. Nanocrystalline films were obtained for the first time ${ }^{83}$

\section{References}

[1] D. Banerjee, J. Williams, Perspectives on Titanium Science and Technology, Acta Materialia 61 (2013) 844-879.

[2] R. Van Noort, Titanium: The implant material of today, Journal of Materials Science 22 (1987) 3801-3811.

[3] Z. Tarnawski, N.-T. T. Kim-Ngan, Hydrogen storage characteristics of Ti- and V-based thin films, Journal of Science: Advanced Materials and Devices 1 (2016) 141-146.

[4] K. Hofmann, B. Spangenberg, M. Luysberg, H. Kurz, Properties of evaporated titanium thin films and their possible application in single electron devices, Thin Solid Films 436 (2003) 168-174. free deposition of [001] preferentially oriented titanium thin film by Kaufman ion-beam source, Thin Solid Films 638 (2017) 57-62.

[6] J.-N. Aoh, C.-L. Chuang, Thermosonic Bonding of Gold Wire onto a Copper Pad with Titanium Thin-Film Deposition, Journal of electronic materials 33 (2004) 290-299.
[5] I. Gablech, O. Caha, V. Svatoš, J. Pekárek, P. Neužil, T. Šikola, Stress- 
[7] J. Yu, J. Wang, B. Lu, Y. Han, Y. Luo, C. Sun, Z. Hao, B. Xiong, 72 L. Wang, H. Li, Characteristics of hexagonal c-oriented titanium film 73 as the template for GaN epitaxy on glass substrate by electron beam 74 evaporation, Thin Solid Films 624 (2017) 160-166.

[8] G. Bernhardt, C. Silvestre, N. LeCursi, S. Moulzolf, D. Frankel, R. Lad, 76 Performance of $\mathrm{Zr}$ and $\mathrm{Ti}$ adhesion layers for bonding of platinum met- 77 allization to sapphire substrates, Sensors and Actuators B: Chemical 7778 (2001) 368-374.

[9] A. Popov, I. Pyshmintsev, S. Demakov, A. Illarionov, T. Lowe, 80 A. Sergeyeva, R. Valiev, Structural and mechanical properties of 81 nanocrystalleve titanium processed by severe plastic deformation, 82 Scripta Materialia 37 (1997) 1089 - 1094

[10] F. E. Wawner, K. R. Lawless, Epitaxial Growth of Titanium Thin Films, 84 Journal of Vacuum Science and Technology 6 (1969) 588-590.

[11] C. Graham, M. M. M. Frances, R. A. Maniyara, Y. Wen, P. Mazumder, 86 V. Pruneri, Nacl substrates for high temperature processing and transfer 87 of ultrathin materials, Scientific reports 10 (2020) 1-7.

[12] J. M. Fink, M. Kalaee, A. Pitanti, R. Norte, L. Heinzle, M. Davanço, 89 K. Srinivasan, O. Painter, Quantum electromechanics on silicon nitride 90 nanomembranes, Nature communications 7 (2016) 1-10.

[13] J. O'Neal, R. Wyatt, F. Leonhard, The deposition of titanium on sapphire in ultrahigh vacuum, Journal of Crystal Growth 7 (1970) 177 - 178.

[14] J. Narayan, B. C. Larson, Domain epitaxy: A unified paradigm for thin 94 film growth, Journal of Applied Physics 93 (2003) 278-285.

[15] V. P. Vlasov, A. E. Muslimov, A. V. Butashin, V. M. Kanevsky, Sapphire 96 evolution of the vicinal (0001) sapphire surface upon annealing in air, 97 Crystallography Reports 61 (2016) 58-62.

[16] J. W. Evans, P. A. Thiel, M. C. Bartelt, Morphological evolution during 99 epitaxial thin film growth: Formation of 2D islands and 3D mounds, 100 Surface Science Reports 61 (2006) 1-128.

[17] J. C. Huang, R. R. Du, C. P. Flynn, Nucleation processes in the growth ${ }_{102}$ of hcp titanium, Physical Review Letters 66 (1991) 341-344.

[18] R Checchetto, Titanium thin film deposition in a deuterium atmosphere, 104 Thin Solid Films 302 (1997) 77-83.

[19] M. J. Jung, K. H. Nam, L. R. Shaginyan, J. G. Han, Deposition of Ti10 thin film using the magnetron sputtering method, Thin Solid Films 435107 (2003) 145-149.

[20] H. Savaloni, A. Taherizadeh, A. Zendehnam, Residual stress and struc-109 tural characteristics in $\mathrm{Ti}$ and $\mathrm{Cu}$ sputtered films on glass substrates at 110 different substrate temperatures and film thickness, Physica B: Con-111 densed Matter 349 (2004) 44-55.

[21] T. Sonoda, A. Watazu, J. Zhu, W. Shi, K. Kato, T. Asahina, Structure113 and mechanical properties of pure titanium film deposited onto $\mathrm{TiNi}_{114}$ shape memory alloy substrate by magnetron DC sputtering, Thin Solid 115 Films 459 (2004) 212-215.

[22] Freibertshauser Pe, McCamont Jw, Electrical properties of Titanium,117 Zirconium and Hafnium films from $3000 \mathrm{~K}$ to $1.30 \mathrm{~K}$, J Vacuum Science 118 \& Technology 6 (1969) 184-187.

[23] G. Dehm, C. Scheu, M. Rühle, R. Raj, Growth and structure of internal 12 $\mathrm{cu} / \mathrm{al} 2 \mathrm{o} 3$ and cu/ti/al2o3 interfaces, Acta Materialia 46 (1998) $759-121$ 772.

[24] M. Huth, C. P. Flynn, Titanium thin film growth on small and large 123 misfit substrates, Applied Physics Letters 71 (1997) 2466-2468.

[25] M. Koyama, S. Arai, S. Suenaga, M. Nakahashi, Interfacial reactions 125 between titanium film and single crystal $\alpha$-A12O3, Journal of Materials126 Science 28 (1993) 830-834.

[26] G. Campbell, T. LaGrange, W. King, J. Colvin, A. Ziegler, N. Browning, 128 H. Kleinschmidt, O. Bostanjoglo, The hep to bcc phase transformation 129 in ti characterized by nanosecond electron microscopy, Proceedings of 130 an International Conference on Solid-Solid Phase Transformations in 131 Inorganic Materials 2 (2005).

[27] L. J. Van der Paw, A method of measuring specific resistivity and hall 133 effect of discs of arbitrary shape, in: Semiconductor Devices: Pioneering ${ }_{134}$ Papers, World Scientific, 1991, pp. 174-182.

[28] P. Scherrer, Bestimmung der inneren Struktur und der Größe von Kol-136 loidteilchen mittels Röntgenstrahlen, Springer Berlin Heidelberg, Berlin, 137 Heidelberg, 1912, pp. 387-409.

[29] The Lattice Constants of High Purity Alpha Titanium, Proceedings of 139 the Physical Society 80 (1962) 783-786.

[30] E. Tal-Gutelmacher, R. Gemma, A. Pundt, R. Kirchheim, Hydrogen behavior in nanocrystalline titanium thin films, Acta Materialia 58 (2010)
3042-3049.

[31] E. Tal-Gutelmacher, A. Pundt, R. Kirchheim, The effect of residual hydrogen on hydrogenation behavior of titanium thin films, Scripta Materialia 62 (2010) 709-712.

[32] F. D. Enck, J. G. Dommel, Behavior of the Thermal Expansion of $\mathrm{NaCl}$ at Elevated Temperatures, Journal of Applied Physics 36 (1965) 839-844.

[33] J. Narayan, B. C. Larson, Domain epitaxy: A unified paradigm for thin film growth, Journal of Applied Physics 93 (2003) 278-285.

[34] J. C. Wurst, J. A. Nelson, Lineal Intercept Technique for Measuring Grain Size in Two-Phase Polycrystalline Ceramics, Journal of the American Ceramic Society 55 (1972) 109.

[35] S. Sadeghi-Khosravieh, K. Robbie, Morphology and crystal texture in tilted columnar micro-structured titanium thin film coatings, Thin Solid Films 627 (2017) 69-76.

[36] M. Vopsaroiu, G. V. Fernandez, M. J. Thwaites, J. Anguita, P. J. Grundy, K. OGrady, Deposition of polycrystalline thin films with controlled grain size, Journal of Physics D: Applied Physics 38 (2005) 490-496.

[37] J. Bae, H. Lee, D. Seo, S. Yun, J. Yang, S. Huh, H. Jeong, J. Noh, Grain size and phase transformation behavior of tini shape-memory-alloy thin film under different deposition conditions, Materials 13 (2020).

[38] J. Estel, H. Hoinkes, H. Kaarmann, H. Nahr, H. Wilsch, On the problem of water adsorption on alkali halide cleavage planes, investigated by secondary ion mass spectroscopy, Surface Science 54 (1976) 393-418.

[39] P. Cabrera-Sanfelix, A. Arnau, G. R. Darling, D. Sanchez-Portal, Water Adsorption and Diffusion on $\mathrm{NaCl}(100)$, The Journal of Physical Chemistry B 110 (2006) 24559-24564.

[40] C. Thompson, R. Carel, Texture development in polycrystalline thin films, Materials Science and Engineering B 32 (1995) 211-219.

[41] V. A. Moskalenko, A. R. Smirnov, A. V. Moskalenko, Cryomechanically obtained nanocrystalline titanium: microstructure and mechanical properties, Low Temperature Physics 35 (2009) 905-907.

[42] Y. Chen, D. M. Bagnall, H.-j. Koh, K.-t. Park, K. Hiraga, Z. Zhu, T. Yao, Plasma assisted molecular beam epitaxy of $\mathrm{ZnO}$ on c-plane sapphire: Growth and characterization, Journal of Applied Physics 84 (1998) 3912-3918.

[43] Y. Wang, S. Wang, S. Zhou, J. Xu, J. Ye, S. Gu, R. Zhang, Q. Ren, Effects of sapphire substrate annealing on $\mathrm{ZnO}$ epitaxial films grown by MOCVD, Applied Surface Science 253 (2006) 1745-1747.

[44] J. Brockman, M. G. Samant, K. P. Roche, S. S. P. Parkin, Substrateinduced disorder in V2O3 thin films grown on annealed c-plane sapphire substrates, Applied Physics Letters 101 (2012) 51606.

[45] T. Hayashi, A. Yamashita, T. Maruno, S. Fölsch, H. Konami, M. Hatano, In-plane ordering of a dibenzo[b,t]phthalocyaninato-zn(ii) thin film due to the atomic step arrays on a sapphire (1012) surface, Journal of Crystal Growth 156 (1995) $245-251$.

[46] G. H. Gilmer, M. H. Grabow, Models of Thin Film Growth Modes 39 (1987) 19-23.

[47] Y. Kakehi, K. Satoh, T. Yotsuya, S. Nakao, T. Yoshimura, A. Ashida, N. Fujimura, Epitaxial growth of $\mathrm{CuScO} 2$ thin films on sapphire aplane substrates by pulsed laser deposition, Journal of Applied Physics 97 (2005) 83535.

[48] D. Rasic, J. Narayan, Epitaxial growth of thin films, in: V. Glebovsky (Ed.), Crystal Growth, IntechOpen, Rijeka, 2019.

[49] D. Gall, The search for the most conductive metal for narrow interconnect lines, Journal of Applied Physics 127 (2020) 050901.

[50] M. E. Day, M. Delfino, J. A. Fair, W. Tsai, Correlation of electrical resistivity and grain size in sputtered titanium films, Thin Solid Films 254 (1995) 285-290.

[51] G. Gould, C. Grahman, E. Grünbaum, L. Moraga, J. Müller, D. C. Larson, Electrical resistivity of epitaxial titanium films, Thin Solid Films 13 (1972) 61-66.

[52] J. Caballero, G. Kremer, L. Moraga, Electrical resistivity of very thin single-crystal titanium films as a function of temperature, Thin Solid Films 117 (1984) 1-8.

[53] E. A. Bel'skaya, E. Y. Kulyamina, Electrical resistivity of titanium in the temperature range from 290 to $1800 \mathrm{~K}$, High Temperature 45 (2007) 785-796. 